\title{
Wisconsin Card Sorting Revisited: Distinct Neural Circuits Participating in Different Stages of the Task Identified by Event- Related Functional Magnetic Resonance Imaging
}

\author{
Oury Monchi, ${ }^{1,2}$ Michael Petrides, ${ }^{2}$ Valentina Petre, ${ }^{1}$ Keith Worsley, ${ }^{1,3}$ and Alain Dagher ${ }^{1}$ \\ ${ }^{1}$ McConnell Brain Imaging Centre and ${ }^{2}$ Cognitive Neuroscience Unit, Montreal Neurological Institute, and ${ }^{3}$ Department of \\ Mathematics and Statistics, McGill University, Montréal, Québec, H3A 2B4 Canada
}

The Wisconsin Card Sorting Task (WCST) has been used to assess dysfunction of the prefrontal cortex and basal ganglia. Previous brain imaging studies have focused on identifying activity related to the set-shifting requirement of the WCST. The present study used event-related functional magnetic resonance imaging (fMRI) to study the pattern of activation during four distinct stages in the performance of this task. Eleven subjects were scanned while performing the WCST and a control task involving matching two identical cards. The results demonstrated specific involvement of different prefrontal areas during different stages of task performance. The middorsolateral prefrontal cortex (area 9/46) increased activity while subjects received either positive or negative feedback, that is at the point when the current information must be related to earlier events stored in working memory. This is consistent with the proposed role of the mid-dorsolateral prefrontal cortex in the monitoring of events in working memory. By contrast, a cortical basal ganglia loop involving the mid-ventrolateral prefrontal cortex (area 47/12), caudate nucleus, and mediodorsal thalamus increased activity specifically during the reception of negative feedback, which signals the need for a mental shift to a new response set. The posterior prefrontal cortex response was less specific; increases in activity occurred during both the reception of feedback and the response period, indicating a role in the association of specific actions to stimuli. The putamen exhibited increased activity while matching after negative feedback but not while matching after positive feedback, implying greater involvement during novel than routine actions.

Key words: basal ganglia; caudate nucleus; fMRl; prefrontal cortex; set-shifting; Wisconsin card sorting
The Wisconsin Card Sorting Task (WCST) has been used to investigate deficits in executive function in humans (Milner, 1963; Nelson, 1976; Stuss et al., 2000). The subject is asked to match test cards to reference cards according to the color, shape, or number of stimuli on the cards. Feedback is provided after each match, enabling the subject to acquire the correct rule of classification. After a fixed number of correct matches, the rule is changed without notice, and the subject must shift to a new mode of classification. Thus, the WCST measures cognitive flexibility, that is the ability to alter a behavioral response mode in the face of changing contingencies (set-shifting).

Patients with lesions of the prefrontal cortex (PFC) are impaired at card sorting (Milner, 1963; Nelson 1976; Stuss et al., 2000). The basal ganglia also play a role in WCST performance as shown by impairments observed in patients with Parkinson's disease (Bowen et al., 1975; Lees and Smith, 1983; Gotham et al., 1988), consistent with the strong anatomical connections between the PFC and basal ganglia (Alexander et al., 1986; Middleton and Strick, 1994). Alexander et al. (1986) proposed the existence of parallel cortical basal ganglia loops, each comprising a specific location in the cortex, basal ganglia, and thalamus. There is

\footnotetext{
Received May 14, 2001; revised July 19, 2001; accepted July 20, 2001.

This work was supported by the Canadian Institutes for Health Research and the International Consortium for Human Brain Mapping, National Institutes of HealthNational Institute of Mental Health. We thank P. Ahad for help with stimulus presentation software, J. Aston and C. Liao for help with data analysis, and A. Charil, A. Evans, P. Neelin, and B. Pike for useful discussions.

Correspondence should be addressed to Dr. Alain Dagher, McConnell Brain Imaging Centre, Montreal Neurological Institute, 3801 University Street, Montréal, Québec, H3A 2B4, Canada. E-mail: alain@bic.mni.mcgill.ca or oury@bic.mni.mcgill.ca. Copyright () 2001 Society for Neuroscience $0270-6474 / 01 / 217733-09 \$ 15.00 / 0$
}

evidence that the nature of the deficit is different in Parkinson's disease than after PFC lesions (Rogers et al., 1998), although the specific roles of PFC and basal ganglia remain unclear.

Functional neuroimaging studies have confirmed the involvement of the PFC in set-shifting (Berman et al., 1995; Nagahama et al., 1996; Goldberg et al., 1998; Konishi et al., 1998, 1999a; Rogers et al., 2000; Nagahama et al., 2001). Basal ganglia involvement has been less evident. Rogers et al. (2000), using positron emission tomography (PET), reported increased activity in the caudate nucleus during an attentional setshifting task only during reversals in the rule of classification, but not during the types of extra-dimensional set-shifts that occur in the WCST. Moreover, the events during set-shifting can be separated into those occurring at the point of receiving negative feedback, indicating that the current set must be changed, and those occurring while the action is performed under the new attentional set. Thus far, brain imaging studies of the WCST have not attempted to differentiate brain activity between these two aspects of set-shifting. In addition, these studies did not separate activity occurring during the moment of receiving positive feedback, indicating that the current set must be maintained, and activity occurring when matching according to the current set. A computational model predicted the involvement of distinct corticostriatal loops during these four stages of the WCST (Monchi et al., 2000). Here, we used mixed-trials event-related functional magnetic resonance imaging (fMRI) to determine the specific location and pattern of activation in the PFC and basal ganglia during these four stages of the WCST. 


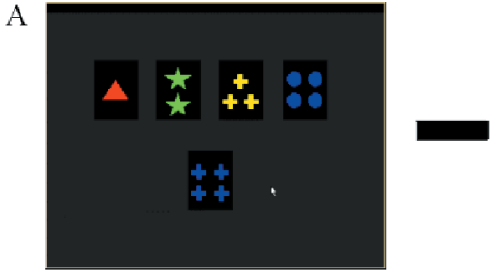

Receiving Negative Feedback

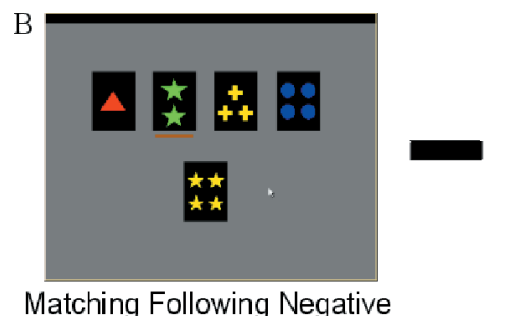

Matching Following Negative Feedback
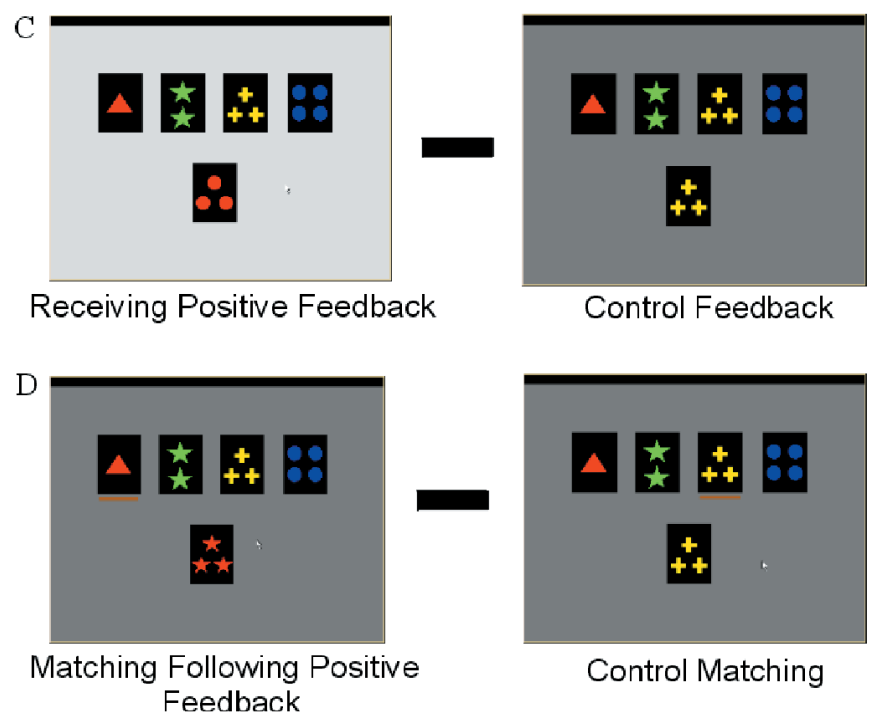

Control Matching

Figure 1. Appearance of the computer monitor during the different events of the WCST task. WCST trials are shown on the left, control trials on the right. The top four cards (on the monitor) are the reference cards, and the bottom card is the test card. During the matching period, the subject chooses the reference card that matches the test card by moving the orange cursor beneath the cards using computer mouse buttons. In the WCST trials, a change in screen brightness during the feedback period indicates a correct or incorrect match. The four subtractions used for data analysis are also illustrated. $A$, Event 1 minus control event $5 ; B$, event 2 minus control event 6 ; $C$, event 3 minus control event $5 ; D$, event 4 minus control event 6 (see Materials and Methods).

\section{MATERIALS AND METHODS}

Subjects. Eleven right-handed subjects (mean age, 24 years; range, 18-31 years; five males, six females) with no history of neurological or psychiatric disorder participated in this study. All subjects gave informed consent to the protocol, which was reviewed and approved by the Research Ethics Committee of the Montreal Neurological Institute.

Cognitive task. A computerized version of the WCST was administered using stimulus presentation software (Media Control Function; Digivox, Montréal, Canada). Subjects were fully trained on the task, using a personal computer, before the scanning session. During scanning, the computer display was projected onto a mirror in the MRI scanner. Throughout this task, four fixed reference cards are present in a row on the top of the screen, displaying one red triangle, two green stars, three yellow crosses, and four blue circles, respectively (Fig. 1). On each trial, a new test card is presented in the middle of the screen below the reference cards. Subjects must then match the test card to one of the reference cards based on the color, shape, or number of the stimuli.
Each trial consists of two periods. The first period starts with the presentation of a new test card. The subject then chooses one of the four reference cards by using two mouse buttons: the left button to move a cursor to point to one of the reference cards, and the right button to confirm the selection. The length of each matching period depends on the subject's response time, which varied between 480 and $3910 \mathrm{msec}$ for this experiment. The second period of each trial starts as soon as the subject has made a selection and consists of feedback conveyed through a change in screen brightness lasting $1900 \mathrm{msec}$. An incorrect classification is indicated by a dark screen during the feedback period (Fig. $1 A$ ), whereas a correct classification is indicated by a bright screen (Fig. 1C).

In addition, there were control trials during which the test card was identical with respect to color, shape, and number to one of the four reference cards. For these control trials, subjects were asked to match the test card to the identical reference card, and the screen maintained its original brightness during the feedback period (Fig. 1A,C). During a scanning session, the subjects performed four types of trials: WCST trials that require matching according to color, shape, or number, and control trials.

Each scanning session consisted of five runs. Blocks of each of the four trial types (the three WCST trials, and the control trial) were presented in random order three times per run. In the WCST blocks, six correct matching responses in a row had to be completed before a change in dimension occurred. The control block consisted of eight trials. The total number of individual trials per run varied with subject performance because it depends on the number of errors.

To study the pattern of activation during the different stages of the WCST, four experimental event periods and two control event periods were defined. These six events were: event 1 , receiving negative feedback, indicating that a shift is required; event 2 , matching after negative feedback, which is the execution of the first match after the set-shift; event 3 , receiving positive feedback, indicating that the current matching criterion must continue to be used; event 4, matching after positive feedback, which is the execution of matching according to the current criterion; event 5, control feedback; event 6 , control matching. Activity in the appropriate control period trials was subtracted from that of the different experimental event periods of the color, shape, and number trials combined to generate the following four contrasts for statistical analysis: (1) receiving negative feedback in the WCST minus control feedback (Fig. 1A); (2) matching after negative feedback in the WCST minus control matching (Fig. $1 B$ ); (3) receiving positive feedback in the WCST minus control feedback (Fig. 1C); and (4) matching after positive feedback in the WCST minus control matching (Fig. 1D).

fMRI scanning. Subjects were scanned using a $1.5 \mathrm{~T}$ Siemens Vision MRI scanner (Siemens AG, Erlangen, Germany). Each scanning session began with a high-resolution T1-weighted three-dimensional volume acquisition for anatomical localization (voxel size, $1 \times 1 \times 1 \mathrm{~mm}^{3}$ ). This was followed by acquisitions of echoplanar $\mathrm{T} 2 *$-weighted images with blood oxygenation level-dependent (BOLD) contrast (TE, $50 \mathrm{msec}$; FA, $90^{\circ}$ ). Functional images were acquired in five runs in a single session. The volumes were acquired continuously every $3.5 \mathrm{sec}$ within each run, and the total number of volumes acquired varied from run to run (from 79 to 113) depending on the subject's performance. Volumes contained 16 slices each of $6 \mathrm{~mm}$ thickness (matrix size, $128 \times 128$ pixels; voxel size, $\left.2.35 \times 2.35 \times 6 \mathrm{~mm}^{3}\right)$. The stimulus presentation and the scanning were synchronized at the beginning of each run.

Data analysis. The first three frames in each run were discarded because the BOLD signal does not reliably reach steady state during those frames. Images from each run were first realigned using the fourth frame as reference. They were then smoothed using a $6 \mathrm{~mm}$ full-width half-maximum (FWHM) isotropic Gaussian kernel. The data analysis was performed using an in-house package (Worsley et al., 2000) (available at http://www.bic.mni.mcgill.ca/users/keith/). The statistical analysis of the fMRI data was based on a linear model with correlated errors. The design matrix of the linear model was first convolved with a difference of two gamma hemodynamic response functions with a mean lag of $5.4 \mathrm{sec}$ timed to coincide with the acquisition of each slice (Glover, 1999). Drift was removed by adding polynomial covariates in the frame times, up to degree three, to the design matrix. Because the response times of the subjects in each trial varied, we were able to obtain BOLD signal at different time points for each type of trial, allowing us to reconstruct the previously defined six events. The correlation structure was modeled as an autoregressive process of degree one (Bullmore et al., 1996). At each voxel, the autocorrelation parameter was estimated from the leastsquares residuals using the Yule-Walker equations, after a bias correc- 
tion for correlation induced by the linear model. The autocorrelation parameter was first regularized by spatial smoothing with a $15 \mathrm{~mm}$ FWHM Gaussian filter, then used to "whiten" the data and the design matrix. The linear model was then re-estimated using least squares on the whitened data to produce estimates of effects and their SEs. Then, the resulting effects and standard effect files were spatially normalized by nonlinear transformation into the standard proportional stereotaxic space of Talairach and Tournoux (1988) using the algorithm of Collins et al. (1994).

In a second step, runs and then subject data were combined using another linear model for the session and subject effects, weighted inversely by the square of their SEs. A random effects analysis was performed by first estimating the ratio of the random effects variance to the fixed effects variance, then regularizing this ratio by spatial smoothing with a $13 \mathrm{~mm}$ FWHM Gaussian filter for the average over runs and a 10 mm FWHM Gaussian filter for the average over subjects. The variance of the effect was estimated then by the smoothed ratio multiplied by the fixed effect variance to achieve higher degrees of freedom.

The resulting $\mathrm{T}$ statistic images were thresholded using the minimum given by a Bonferroni correction and random field theory to account for multiple comparisons (Worsley et al., 1996). The threshold was calculated on the basis of an estimated gray matter volume of $600 \mathrm{~cm}^{3}$. For a single voxel, this yields a threshold of $\mathrm{t}>4.70$, which corresponds to $p<$ 0.05 corrected. For statistical peaks below that threshold, significance was also assessed on the basis of the spatial extent of the cluster of contiguous voxels with $p<0.05$ corrected using the method of Friston et al. (1995).

\section{RESULTS}

All 11 subjects completed 45 WCST conditions (five runs, nine conditions per run). They made on average 0.45 perseverative errors and 6.65 nonperseverative, or set-loss, errors during the scanning session. They made an average of 52.87 incorrect classifications after shifts in condition. We compared the BOLD signal that was obtained during the trials requiring matching according to color, shape, and number (combined) with that obtained during the corresponding periods in the control trials. As predicted, a network of structures involving the PFC, basal ganglia, and thalamus showed relatively greater activity during different stages of WCST performance than during corresponding control conditions (see Tables 1-4).

\section{Receiving negative feedback}

When activity during the period of receiving negative feedback (event 1) was compared with the control feedback condition (event 5) (Table 1), there were significant activity increases, bilaterally, in the mid-dorsolateral PFC (areas 46, 9/46) (Fig. 2A), in the mid-ventrolateral PFC (area 47/12) (Fig. 2B), and in the posterior PFC at the most caudal part of the inferior frontal sulcus, namely at the junction of rostral area 6 with area $8 \mathrm{~A}$ and area 44 (Fig. 2C). There were also bilateral increases of activity in the caudate nucleus and the dorsal thalamus (Table 1, Fig. 2D). These structures make up the prefrontal cortical basal ganglia loops (Alexander et al., 1986). Significant activation was also found, bilaterally, in the rostral anterior cingulate cortex (area 32), lateral premotor cortex (area 6), posterior parietal cortex (areas 7 and 40), and prestriate cortex (area 19). Relative to the control, there was a reduction in the BOLD signal in the left medial frontal cortex (area 10), left motor cingulate region (area 24), left motor cortex (area 4), and bilateral putamen and posterior parietal cortex during the reception of negative feedback (Table 1).

\section{Matching after negative feedback}

When the period of matching after negative feedback (event 2) was compared with the control matching period (event 6) (Table 2), the BOLD signal was greater in the left putamen (Fig. 3) and left posterior PFC (Fig. 2C). There were also increases in activity in the posterior parietal cortex (area 7), prestriate cortex (area 19), and right lateral premotor cortex (area 6). A reduction in BOLD signal in the right restroplenial cortex (area 30) was also observed.

\section{Receiving positive feedback}

When activity during the period of receiving positive feedback (event 3) was compared with the control feedback condition (event 5) (Table 3), there were significant increases in BOLD signal in the right mid-dorsolateral PFC (areas 46, 9/46), posterior PFC (area 8), restroplenial cortex (area 30), and left posterior parietal cortex (area 40). A reduction in the BOLD signal in the lateral premotor cortex (area 6) was also observed.

\section{Matching after positive feedback}

Activity during matching after positive feedback (event 4) compared with the control matching condition (event 6) (Fig. 1D) yielded only three significant positive peaks, namely the lateral premotor cortex (area 6), bilaterally, and the left posterior parietal cortex (area 7) (Table 4). There was a reduction in BOLD signal in the right restroplenial cortex (area 30) and the right posterior parietal cortex (area 40).

\section{Receiving negative feedback relative to receiving positive feedback}

To understand further the specificity of involvement of the different areas of the PFC and the basal ganglia in WCST performance, the change in BOLD signal was also examined by subtracting activity during the period of receiving positive feedback (event 3 ) from the period of receiving negative feedback (event 1) (Table 5). Significantly greater BOLD signal was found bilaterally in the mid-ventrolateral PFC (area 47/12), the caudate nucleus, and the mediodorsal thalamus (Fig. 2D). These structures make up a prefrontal cortical basal ganglia loop originating in the mid-ventrolateral PFC (Alexander et al., 1986). Significant activation was also found using this contrast in the right prestriate cortex (area 19), the left lateral premotor cortex (area 6), and the right posterior parietal cortex (area 7). The reverse subtraction, receiving positive feedback minus receiving negative feedback, did not give rise to any significant differences.

\section{DISCUSSION}

We used event-related fMRI to dissociate activity related to shifting versus maintaining an attentional set and to receiving feedback versus selecting the appropriate action during the performance of the WCST. First, there was a dissociation between the mid-ventrolateral and mid-dorsolateral prefrontal areas. Although both showed increased activity during set-shifting, only the mid-dorsolateral PFC also showed increased activity during set maintenance. Second, the role in set-shifting of the posterior lateral PFC (Konishi et al., 1998, 1999a) was clarified and shown to be different from that of the mid-dorsolateral and midventrolateral prefrontal areas. Third, consistent with clinical studies, the caudate and putamen were found to be involved in performance of the task.

\section{Differential prefrontal activity}

After matching a card, the subject receives feedback that must be related to information in working memory about earlier trials to decide whether to maintain or shift the current attentional set. Thus, responding to either negative or positive feedback involves monitoring the contents of working memory. In the present study, receiving either type of feedback compared with the control 
Table 1. Receiving negative feedback (event 1 ) minus control feedback (event 5)

Anatomical area

Positive peaks

Mid-dorsolateral prefrontal cortex (areas 46, 9/46)

Left

Right

Mid-ventrolateral prefrontal cortex (area 47/12)

Left

Right

Anterior cingulate cortex (area 32 rostral)

Left

Right

Posterior prefrontal cortex (junction of areas $6,8,44$ )

Left

Right

(Area 8) Right

Lateral premotor cortex (area 6)

Left

Right

Posterior parietal cortex (area 40)

Left

Right

Posterior parietal cortex (area 7)

Left

Right

Prestriate cortex (area 19)

Left

Right

Mediodorsal thalamus

Left

Right

Caudate nucleus

Left

Right

Negative peaks

Medial frontal gyrus (area 10)

Left

Motor cingulate region (area 24)

Left

Motor cortex (area 4)

Left

Posterior parietal cortex (area 40)

Left

Right

Putamen

Left

Right
Stereotaxic coordinates

$t$ stat

$p$ value

$-503023$

423028

$-3620-2$

34222

$-44-4646$

$-34-5244$

$34-4644$

$-30-6448$

$-14-7456$

$30-5652$

$16-7644$

$-26-6834$

$-26-8630$

$31-6628$

$35-7424$

$-8-2210$

$6-1610$

$10-104$

$8-108$

$-16144$

12123

$-125210$

$0-640$

$-10-1244$

$-32-2460$

$-38-3258$

$-30-1662$

$-34-2858$

$-64-3832$

$62-2826$

$50-2820$

$-26-106$

$28-124$
5.26

4.15

7.65

6.44

5.27

5.26

5.15

4.92

4.98

4.47

6.21

5.47

4.82

4.94

$<0.01$

$<0.05^{*}$

$<0.001$

$<0.001$

$<0.01$

$<0.01$

$<0.01$

$<0.05$

$<0.05$

$<0.05$ *

$<0.001$

$<0.001$

$<0.05$

$<0.01$

$<0.01$

$<0.01$

$<0.001$

$<0.001$

$<0.001$

$<0.001$

$<0.001$

$<0.001$

$<0.001$

$<0.001$

$<0.05$

$<0.05$

$<0.05$

$<0.05$

$<0.05^{*}$

$<0.05^{*}$

3.31

$-5.60$

$<0.001$

$-5.60$

$<0.001$

$-4.71$

$<0.05$

$-5.28$

$<0.01$

$-4.94$

$<0.05$

$-4.74$

$<0.05$

$-5.29$

$<0.01$

$-4.83$

$<0.05$

$<0.05$

$<0.05$

$-4.65$

$<0.01$

$-5.32$

$<0.01$
$<0.05$

All $p$ values corrected for multiple comparisons. *Significant by cluster size analysis. 


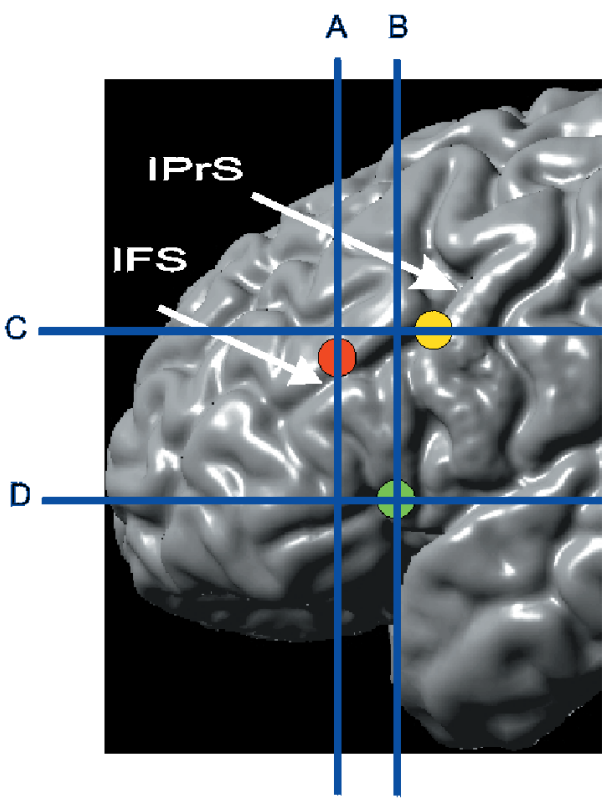

T
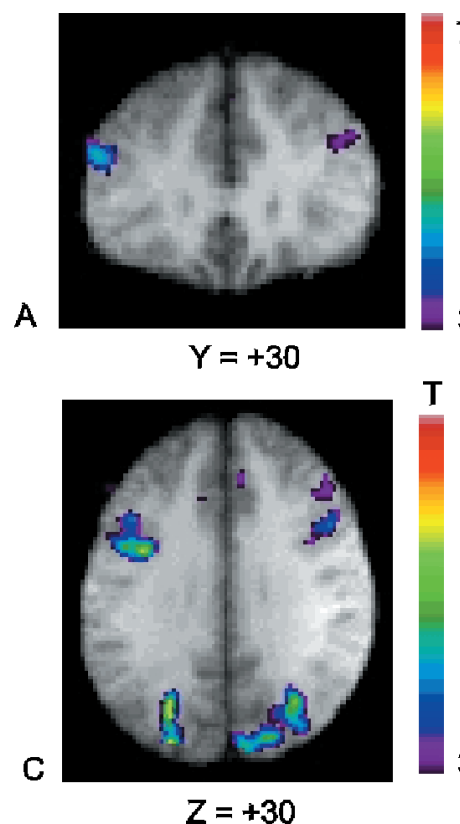

7.75

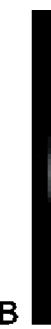

$3.75 \mathrm{~B}$

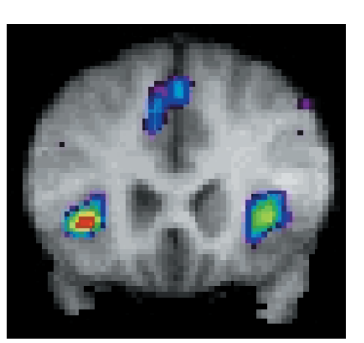

$Y=+22$

7.75

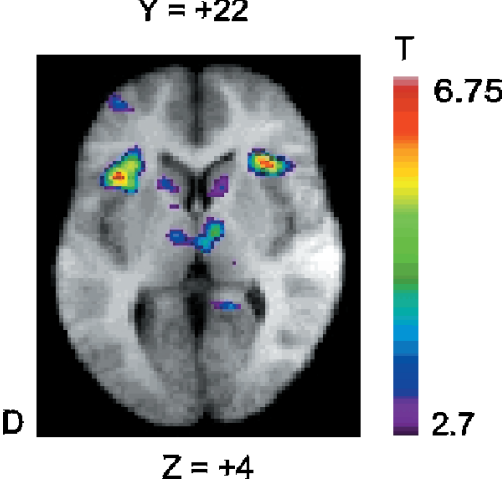

T

7,75

6.75

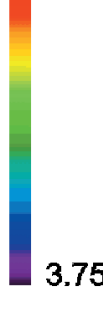

3.75

$Z=+4$

Figure 2. Location of prefrontal peaks. The top left panel displays the sites of the main prefrontal areas identified in the experiment on a cortical surface rendering of an MRI in standard stereotaxic space. The vertical blue lines indicate the anteroposterior level of the coronal sections in $A$ and $B$. The horizontal blue lines indicate the dorsoventral level of the sections displayed in $C$ and $D$. The focus in the mid-dorsolateral PFC is indicated by the red circle, in mid-ventrolateral PFC by the green circle, and in posterior PFC by the yellow circle. A, Coronal section through the mid-dorsolateral PFC peak at $\mathrm{Y}=+30 \mathrm{~mm}$. $B$, Coronal section through the mid-ventrolateral PFC peak at $\mathrm{Y}=+22 \mathrm{~mm}$. $C$, Horizontal section through the posterior PFC peak at $\mathrm{Z}=+30 \mathrm{~mm}$. $D$, Horizontal section through the mid-ventrolateral PFC peak at $\mathrm{Z}=+4 \mathrm{~mm}$. Note also caudate and thalamus activation. All activation peaks shown here occurred during receiving negative feedback (event 1) minus control feedback (event 5). Some of these PFC peaks also occur selectively in other subtractions, permitting their functional dissociation (see Discussion and Table 6). The anatomical MRI images shown in $A-D$ are the average of the T1 acquisitions of the 11 subjects transformed into stereotaxic space. The color scale represents the T statistic. IFS, Inferior frontal sulcus; $\operatorname{IPr} S$, inferior precentral sulcus.

\begin{tabular}{|c|c|c|c|}
\hline Anatomical area & $\begin{array}{l}\text { Stereotaxic } \\
\text { coordinates }\end{array}$ & $t$ stat & $p$ value \\
\hline \multicolumn{4}{|l|}{ Positive peaks } \\
\hline $\begin{array}{l}\text { Posterior prefrontal cortex } \\
\text { (areas } 6,8,44), \text { left }\end{array}$ & -361426 & 4.18 & $<0.05^{*}$ \\
\hline $\begin{array}{l}\text { Lateral premotor cortex } \\
\quad \text { (area 6), right }\end{array}$ & $20-1150$ & 3.54 & $<0.05^{*}$ \\
\hline $\begin{array}{l}\text { Posterior parietal cortex } \\
\quad \text { (area 7), left }\end{array}$ & $\begin{array}{lll}-24 & -68 & 44 \\
-28 & -60 & 50\end{array}$ & $\begin{array}{l}6.28 \\
5.49\end{array}$ & $\begin{array}{l}<0.001 \\
<0.01\end{array}$ \\
\hline Prestriate cortex (area 19) & $40-6414$ & 4.72 & $<0.05$ \\
\hline Putamen, left & $-26-104$ & 4.80 & $<0.05$ \\
\hline \multicolumn{4}{|l|}{ Negative peaks } \\
\hline $\begin{array}{l}\text { Posterior cingulate region } \\
\text { (restroplenial cortex, } \\
\text { area 30), right }\end{array}$ & $8-4826$ & -5.16 & $<0.01$ \\
\hline
\end{tabular}

All $p$ values corrected for multiple comparisons. *Significant by cluster size analysis.

condition was associated with increased signal in the middorsolateral PFC (area 9/46) (Tables 1, 3, 6; Fig. 2A). This area has previously been shown to be involved in monitoring information in working memory (Petrides et al., 1993a, 1993b), a finding that has been replicated several times with various types of stimulus material (for review, see Owen, 1997; Petrides, 2000). Moreover, in monkeys, lesions confined to the mid-dorsolateral

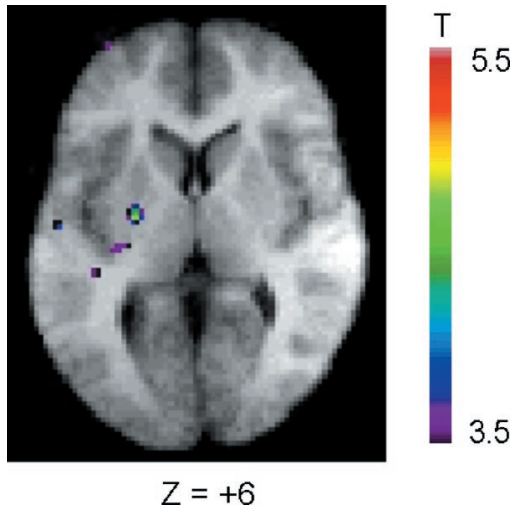

Figure 3. Putamen activation while matching after a set-shift. Activation map of the subtraction: matching after positive feedback (event 2) minus control matching (event 6). Horizontal section at $\mathrm{Z}=+6 \mathrm{~mm}$. The anatomical MRI is the average of the T1 acquisitions of the 11 subjects transformed into stereotaxic space.

PFC yield a severe and selective impairment in the monitoring of working memory without affecting the maintenance of information in memory per se (Petrides, 1991, 1995).

In contrast to the mid-dorsolateral prefrontal region, the midventrolateral prefrontal area 47/12 was significantly involved while receiving negative feedback (Tables $1,5,6$; Fig. $2 B, D$ ), that is, at the point necessitating a set-shift, but not while receiving positive feedback. In the monkey, shifting from a previously relevant to a new response mode is impaired by lesions of the inferior prefrontal convexity (Iversen and Mishkin, 1970). This part of the PFC is 


\begin{tabular}{|c|c|c|c|}
\hline Anatomical area & $\begin{array}{l}\text { Stereotaxic } \\
\text { coordinates }\end{array}$ & $t$ stat & $p$ value \\
\hline \multicolumn{4}{|l|}{ Positive peaks } \\
\hline $\begin{array}{l}\text { Mid-dorsolateral prefrontal cortex } \\
\text { (area 46, 9/46), right }\end{array}$ & 483622 & 4.70 & $<0.05$ \\
\hline $\begin{array}{l}\text { Posterior prefrontal cortex } \\
\text { (area 8), right }\end{array}$ & 381642 & 5.02 & $<0.05$ \\
\hline $\begin{array}{l}\text { Posterior cingulate region } \\
\text { (restroplenial cortex, area 30), } \\
\text { right }\end{array}$ & $8-4628$ & 4.70 & $<0.05$ \\
\hline \multicolumn{4}{|l|}{ Posterior parietal cortex (area } \\
\hline 40$),$ left & $-42-6440$ & 4.51 & $<0.05^{*}$ \\
\hline \multicolumn{4}{|l|}{ Negative peaks } \\
\hline $\begin{array}{l}\text { Lateral premotor cortex (area 6), } \\
\text { right }\end{array}$ & $26-654$ & -5.06 & $<0.01$ \\
\hline
\end{tabular}

All $p$ values corrected for multiple comparisons. *Significant by cluster size analysis.

\begin{tabular}{llll}
\hline $\begin{array}{l}\text { Table 4. Matching after positive feedback (event 4) minus control } \\
\text { matching (event 6) }\end{array}$ & & \\
& $\begin{array}{l}\text { Stereotaxic } \\
\text { coordinates }\end{array}$ & $t$ stat & $p$ value \\
Anatomical area & & & \\
\hline Positive peaks & & & \\
$\quad$ Lateral premotor cortex (area 6) & $-50-642$ & 4.70 & $<0.05$ \\
$\quad$ Left & $24-654$ & 4.51 & $<0.05^{*}$ \\
$\quad$ Right & & & \\
Posterior parietal cortex (area 7) & & & \\
$\quad$ Left & & & \\
Negative peaks & & & \\
Posterior cingulate region (re- & & & \\
$\quad$ stroplenial cortex, area 30) & $8-4628$ & -7.35 & $<0.001$ \\
$\quad$ Right & $4-3238$ & -4.68 & $<0.05$ \\
$\quad$ & $42-4830$ & -6.96 & $<0.001$ \\
Posterior parietal cortex (area 40) & & & \\
$\quad$ Right & $62-4034$ & -4.94 & $<0.05$ \\
&
\end{tabular}

All $p$ values corrected for multiple comparisons. *Significant by cluster size analysis.

architectonically and topographically comparable with the human ventrolateral PFC (Petrides and Pandya, 1994). It can therefore be inferred that the mid-ventrolateral prefrontal activity seen here is selectively related to the cognitive events involved in shifting attentional set. This difference in mid-dorsolateral versus mid-ventrolateral PFC involvement in the WCST is consistent with a theoretical position that posits a major difference in the role of the mid-dorsolateral PFC, which is necessary for the monitoring of information in working memory, as opposed to the mid-ventrolateral PFC, which is considered to be involved in more basic executive processing, such as the active comparison of stimuli held in working memory (Petrides, 1994, 1996).

The neural activity during the reception of feedback could be a result of the response to the rewarding or punishing values of the feedback stimulus as well as to the act of mentally shifting set. Previous fMRI studies have implicated the orbitofrontal cortex (OFC) and ventral striatum in the reception of reward and punishment. For example, the passive reception of monetary gains or losses activated these regions, but not the mid-ventrolateral PFC or dorsal striatum (Breiter et al., 2001). In two other fMRI
Table 5. Receiving negative feedback (event 1) minus receiving positive feedback (event 3)

\begin{tabular}{|c|c|c|c|}
\hline Broadmann's area & $\begin{array}{l}\text { Stereotaxic } \\
\text { coordinates }\end{array}$ & $t$ stat & $p$ value \\
\hline \multicolumn{4}{|c|}{$\begin{array}{l}\text { Mid-ventrolateral prefrontal cortex } \\
\text { (area 47/12) }\end{array}$} \\
\hline Left & -34220 & 5.65 & $<0.001$ \\
\hline Right & 32244 & 4.98 & $<0.05$ \\
\hline \multicolumn{4}{|c|}{ Posterior parietal cortex (area 7) } \\
\hline Right & $18-5652$ & 4.85 & $<0.01$ \\
\hline \multicolumn{4}{|c|}{ Prestriate cortex (area 19) } \\
\hline \multirow[t]{2}{*}{ Right } & $36-7424$ & 5.19 & $<0.01$ \\
\hline & $28-7236$ & 5.10 & $<0.01$ \\
\hline \multicolumn{4}{|l|}{ Caudate } \\
\hline Left & -884 & 3.44 & $<0.05^{*}$ \\
\hline Right & 464 & 3.30 & $<0.05^{*}$ \\
\hline \multicolumn{4}{|l|}{ Thalamus } \\
\hline Left & $-10-1812$ & 3.60 & $<0.05^{*}$ \\
\hline Right & $6-144$ & 3.55 & $<0.05^{*}$ \\
\hline
\end{tabular}

All $p$ values corrected for multiple comparisons. *Significant by cluster size analysis.

studies, the reception of reward or penalty during simple tasks not requiring set-shifting or learning also activated the lateral $\mathrm{OFC}$ but not the mid-ventrolateral or dorsolateral PFC (Elliott et al., 2000; Zalla et al., 2000). However, a fMRI study of monetary rewards and losses during a reversal learning task showed involvement of both lateral OFC and mid-ventrolateral PFC after negative feedback, which signaled both a monetary loss and a need for a shift in strategy (O'Doherty et al., 2001). These findings suggest that the OFC responds to emotionally salient feedback, whereas the ventrolateral PFC is only involved if it is necessary to plan a response to the negative feedback. We suggest that the lack of OFC involvement in our study is attributable to the lack of emotional salience of the feedback in the WCST compared with these other paradigms.

Previous imaging studies of the WCST demonstrated increased activity in a posterior lateral prefrontal area during set-shifting located at the most posterior part of the inferior frontal sulcus (Konishi et al., 1998, 1999a). This activity focus was also observed in the present study, but it could be clearly dissociated from activity in the mid-dorsolateral and mid-ventrolateral PFC. This posterior prefrontal region, at the junction of areas $44,8 \mathrm{~A}$, and rostral 6 , corresponds to the periarcuate region of the macaque monkey cortex (Petrides and Pandya, 1994). Lesions confined to the periarcuate region cause impairment in the selection of alternative responses on the basis of conditional rules (i.e., select response $\mathrm{X}$ when stimulus $\mathrm{A}$, but response $\mathrm{Y}$ when stimulus $\mathrm{B}$ ) in monkeys (Halsband and Passingham, 1982; Petrides, 1982, 1987) and humans (Petrides, 1985, 1990). Importantly, this conditional learning deficit can be dissociated from the impairment in monitoring of working memory caused by mid-dorsolateral prefrontal lesions (Petrides, 1987).

In the present study, activation of this posterior lateral prefrontal region was found during the reception of both positive and negative feedback, as well as during matching after negative feedback. Thus, activation of this region, unlike the middorsolateral and mid-ventrolateral cortex, was seen during the actual matching responses. This finding, taken in conjunction with those obtained from lesion studies in monkeys and humans, suggests that the posterior lateral prefrontal region may be in- 


\begin{tabular}{lllll}
\hline \multicolumn{5}{l}{ Table 6. Frontal and basal ganglia activation during the WCST } \\
& $\begin{array}{l}\text { Receiving negative } \\
\text { feedback }\end{array}$ & $\begin{array}{l}\text { Matching after } \\
\text { negative feedback }\end{array}$ & $\begin{array}{l}\text { Receiving positive } \\
\text { feedback }\end{array}$ & $\begin{array}{l}\text { Matching after positive } \\
\text { feedback }\end{array}$ \\
\hline DLPFC & B & - & R & - \\
VLPFC & B & - & - & - \\
pPFC & B & L & R & - \\
ACC & B & - & - & - \\
Caudate & B & - & - & - \\
Putamen & - & L & - & -
\end{tabular}

Each box represents the presence of increased activity relative to the appropriate control condition. B, Bilateral; R, right; L, left; DLPFC, dorsolateral prefrontal cortex; VLPFC, ventrolateral prefrontal cortex; pPFC, posterior prefrontal cortex; ACC, anterior cingulate cortex.

volved in the selection of the appropriate response on the basis of the currently relevant rule rather than in the establishment or maintenance of the rule.

Other imaging studies are consistent with this notion. When Konishi et al. (1999a) modified the WCST by explicitly informing subjects of the dimensional shifts, they still detected transient BOLD activity bilaterally in the posterior prefrontal area at the time of the shifts. Thus, this area is involved in establishing the correct conditional response even when the rule does not have to be determined by trial and error. Nagahama et al. (2001) also found a dissociation between posterior PFC and mid-dorsolateral PFC when comparing a reversal task to a set-shifting task designed so that the same responses were made to the same stimuli, but according to different rules. The posterior PFC was involved in both conditions, but the mid-dorsolateral PFC was only activated in the set-shifting task, suggesting that only the latter area played a role in higher-level control of response set. Other imaging studies have shown involvement of the same posterior PFC area in the inhibition of incorrect responses (Taylor et al., 1997; Konishi et al., 1999b) and in conditional visuomotor mapping (Toni and Passingham, 1999).

We also observed significant activation in the rostral anterior cingulate cortex bilaterally only during reception of negative feedback. This is consistent with its proposed role in detecting the occurrence of conflict between prediction and outcome (Berns et al., 1997; Carter et al., 1998) and in situations in which an action must be selected from among competing alternatives (Paus et al., 1993). Finally, the posterior parietal cortex was activated during all phases of the task. Involvement of this area has been described in previous PET studies of the WCST (Berman et al., 1995; Nagahama et al., 1996; Goldberg et al., 1998) and may account for impairments on the task seen in patients with focal parietal lesions (Anderson et al., 1991).

\section{Cortical basal ganglia loops and attentional set}

There was significantly greater signal in the caudate and putamen during the WCST than the control task. The activation was only detected in negative feedback trials, consistent with other imaging studies showing greater striatal activity during novel tasks (Berns et al., 1997; Jueptner et al., 1997). Our results may appear to contradict the PET study of Rogers et al. (2000), who failed to find ventrolateral PFC or striatal activation related to shifting attention to a new stimulus dimension. This may be because the transient neural events associated with shifting dimension once during a $90 \mathrm{sec}$ PET acquisition had relatively little effect on the total measured radioactivity.

In our study, the activity in the caudate and putamen paralleled that in the frontal cortex. Comparing the reception of negative feedback to control feedback showed a bilateral increase in activity in the caudate nucleus and mediodorsal thalamus, as well as in the mid-dorsolateral and mid-ventrolateral PFC. These prefrontal and subcortical structures form the "cognitive" cortical basal ganglia loop, as originally proposed by Alexander et al. (1986), who also suggested that the function of each loop was linked to the function of the cortical area that belongs to it. Interestingly, when matching after negative feedback was compared with control matching, there was significant activation in the left putamen and left posterior lateral PFC, structures forming one of the motor loops of Alexander et al. (1986). Note that in control matching, the subjects made the same movements as when matching after negative feedback. Thus, the observed activation reflects the involvement of the putamen and posterior lateral PFC in performing an action according to a behavioral rule. By contrast, the caudate and mid-dorsalateral and mid-ventrolateral PFC were activated during the setting of the rule, but not during the actual choice of action according to that rule.

This pattern of involvement of the basal ganglia is consistent with single cell recordings in animals. During visuomotor tasks, caudate neurons are most active during preparation for movement, whereas putamen neurons fire mostly in relation to movement (Rolls, 1994). Moreover, the firing of these neurons is usually context-dependent (Rolls, 1994; Houk and Wise, 1995), meaning that a neuron will fire in relation to a particular cue or behavioral response only within the context of a certain task. This context dependency is a key feature of attentional set and is also observed in PFC (Wise et al., 1996).

There is evidence that striatal activity is modulated by selective attention. Boussaoud and Kermadi (1997) performed single-cell recordings in the striatum of monkeys during a conditional visuomotor task. They found large numbers of cells responding to cues that reoriented spatial attention, even before the specification of the appropriate motor response. PET experiments in humans confirm the role of the basal ganglia in attention (Vandenberghe et al., 1996; Koski et al., 1999), and patient studies have demonstrated impaired control of visual attention in Parkinson's disease (Wright et al., 1990; Yamada et al., 1990) and after vascular lesions of the basal ganglia (Sakashita, 1991).

The involvement of the caudate and putamen reported here possibly explains the deficit on the WCST reported in Parkinson's disease (Bowen et al., 1975; Lees and Smith, 1983; Gotham et al., 1988). The finding of striatal activation in the WCST is consistent with the theory that the basal ganglia are involved in selecting the relevant action among competing motor responses (Mink and Thach, 1993). We propose, furthermore, that the basal ganglia are particularly important in determining 
attentional set and guiding action in response to behavioral rules, in conjunction with the PFC.

\section{REFERENCES}

Alexander GE, DeLong MR, Strick PL (1986) Parallel organization of functionally segregated circuits linking basal ganglia and cortex. Annu Rev Neurosci 9:357-381.

Anderson SW, Damasio H, Jones RD, Tranel D (1991) Wisconsin Card Sorting Test performance as a measure of frontal lobe damage. J Clin Exp Neuropsychol 13:909-922.

Berman KF, Ostrem JL, Randolph C, Gold J, Goldberg TE, Coppola R, Carson RE, Herscovitch P, Weinberger DR (1995) Physiological activation of a cortical network during performance of the Wisconsin Card Sorting Test: a positron emission tomography study. Neuropsychologia 33:1027-1046.

Berns GS, Cohen JD, Mintun MA (1997) Brain regions responsive to novelty in the absence of awareness. Science 276:1272-1275.

Boussaoud D, Kermadi I (1997) The primate striatum: neuronal activity in relation to spatial attention versus motor preparation. Eur J Neurosci 9:2152-2168.

Bowen FP, Kamienny RS, Burns MM, Yahr M (1975) Parkinsonism: effects of levodopa treatment on concept formation. Neurology 25:701-704.

Breiter HC, Aharon I, Kahneman D, Dale A, Shizgal P (2001) Functional imaging of neural responses to expectancy and experience of monetary gains and losses. Neuron 30:619-639.

Bullmore ET, Brammer MJ, Williams SCR, Rabe-Hesketh S, Janot N, David AS, Mellers JDC, Howard R, Sham P (1996) Statistical methods of estimation and inference for functional MR image analysis. Magn Reson Med 35:261-277.

Carter CS, Braver TS, Barch DM, Botvinick MM, Noll D, Cohen JD (1998) Anterior cingulate cortex, error detection, and the online monitoring of performance. Science 280:747-749.

Collins DL, Neelin P, Peters TM, Evans AC (1994) Automatic 3D intersubject registration of MR volumetric data in standardized talairach space. J Comput Assist Tomogr 18:192-205.

Elliott R, Friston KJ, Dolan RJ (2000) Dissociable neural responses in human reward systems. J Neurosci 20:6159-6165.

Friston KJ, Holmes AP, Worsley KJ, Poline JB, Frith CD, Frackowiak RSJ (1995) Statistical parametric maps in functional imaging: a general linear approach. Hum Brain Mapp 2:189-210.

Glover GH (1999) Deconvolution of impulse response in event-related BOLD fMRI. NeuroImage 9:416-429.

Goldberg TE, Berman KF, Fleming K, Ostrem J, Van Horn JD, Esposito G, Mattay VS, Gold JM, Weinberger DR (1998) Uncoupling cognitive workload and prefrontal cortical physiology: a PET rCBF study. NeuroImage 7:296-303.

Gotham AM, Brown RG, Marsden CD (1988) "Frontal” cognitive function in patients with Parkinson's disease "on" and "off" levodopa. Brain 111:299-321.

Halsband U, Passingham R (1982) The role of premotor and parietal cortex in the direction of action. Brain Res 240:368-372.

Houk JC, Wise SP (1995) Distributed modular architectures linking basal ganglia, cerebellum, and cerebral cortex: their role in planning and controlling action. Cereb Cortex 5:95-110.

Iversen SD, Mishkin M (1970) Perseverative interference in monkeys following selective lesions of the inferior prefrontal convexity. Exp Brain Res 11:376-386.

Jueptner M, Frith CD, Brooks DJ, Frackowiak RS, Passingham RE (1997) Anatomy of motor learning. II. Subcortical structures and learning by trial and error. J Neurophysiol 77:1325-1337.

Konishi S, Nakajima K, Uchida I, Kameyama M, Nakahara K, Sekihara K, Miyashita Y (1998) Transcient activation of inferior prefrontal cortex during cognitive set shifting. Nat Neurosci 1:80-84.

Konishi S, Kawazu M, Uchida I, Kikyo H, Asakura I, Miyashita Y (1999a) Contribution of working memory to transient activation in human inferior prefrontal cortex during performance of the Wisconsin Card Sorting Test. Cereb Cortex 9:745-753.

Konishi S, Nakajima K, Uchida I, Kikyo H, Kameyama M, Miyashita Y (1999b) Common inhibitory mechanism in human inferior prefrontal cortex revealed by event-related functional MRI. Brain 122:981-991.

Koski L, Paus T, Hofle N, Petrides M (1999) Increased blood flow in the basal ganglia when using cues to direct attention. Exp Brain Res 129:241-246.

Lees AJ, Smith E (1983) Cognitive deficits in the early stages of Parkinson's disease. Brain 106:257-270.

Middleton FA, Strick PL (1994) Anatomical evidence for cerebellar and basal ganglia in higher cognitive functions. Science 266:458-461.

Milner B (1963) Effects of brain lesions on card sorting. Arch Neurol 9:90-100.

Mink JW, Thach WT (1993) Basal ganglia intrinsic circuits and their role in behavior. Curr Opin Neurobiol 3:950-957.
Monchi O, Taylor JG, Dagher A (2000) A neural model of working memory processes in normal subjects, Parkinson's disease and schizophrenia for fMRI design and predictions. Neural Netw 13:953-973.

Nagahama Y, Fukuyama H, Yamauchi H, Matsuzaki S, Konishi J, Shibasaki H, Kimura J (1996) Cerebral activation during performance of a card sorting test. Brain 119:1667-1675.

Nagahama Y, Okada T, Katsumi Y, Hayashi T, Yamauchi H, Oyanagi C, Konishi J, Fukuyama H, Shibasaki H (2001) Dissociable mechanisms of attentional control within the human prefrontal cortex. Cereb Cortex 11:85-92.

Nelson H (1976) A modified card sorting response sensitive to frontal lobe defects. Cortex 12:313-324.

O’Doherty J, Kringelbach ML, Rolls ET, Hornak J, Andrews C (2001) Abstract reward and punishment representations in the human orbitofrontal cortex. Nat Neurosci 4:95-102.

Owen AM (1997) The functional organization of working memory processes within human lateral frontal cortex: the contribution of functional neuroimaging. Eur J Neurosci 9:1329-1339.

Paus T, Petrides M, Evans AC, Meyer E (1993) Role of the human anterior cingulate cortex in the control of oculomotor, manual, and speech responses: a positron emission tomography study. J Neurophysiol 70:453-469.

Petrides M (1982) Motor conditional associative-learning after selective prefrontal lesions in the monkey. Behav Brain Res 5:407-413.

Petrides M (1985) Deficits on conditional associative-learning task after frontal- and temporal-lobe lesions in man. Neuropsychologia 23:601-614.

Petrides M (1987) Conditional learning and the primate frontal cortex. In: The frontal lobes revisited (Perecman E, ed), pp 91-108. New York: IRBN.

Petrides M (1990) Nonspatial conditional learning impaired in patients with unilateral frontal but not unilateral temporal lobe excisions. Neuropsychologia 28:137-149.

Petrides M (1991) Monitoring of selections of visual stimuli and the primate frontal cortex. Proc R Soc Lond B Biol Sci 246:293-298.

Petrides M (1994) Frontal lobes and working memory: evidence from investigations of the effects of cortical excisions in nonhuman primates. In: Handbook of neuropsychology, Vol 9 (Boller F, Grafman J, eds), pp 59-81. Amsterdam: Elsevier.

Petrides M (1995) Impairments on nonspatial self-ordered and externally ordered working memory tasks after lesions of the mid-dorsal part of the lateral frontal cortex in the monkey. J Neurosci 15:359-375.

Petrides M (1996) Specialized systems for the processing of mnemonic information within the primate frontal cortex. Philos Trans R Soc Lond B Biol Sci 351:1455-1462.

Petrides M (2000) Mapping prefrontal cortical systems for the control of cognition. In: Brain mapping: the systems (Toga WA, Mazziotta JC, eds), pp 159-176. San Diego: Academic.

Petrides M, Pandya DN (1994) Comparative architectonic analysis of the human and the macaque frontal cortex. In: Handbook of neuropsychology, Vol 9 (Boller F, Grafman J, eds), pp 17-58. Amsterdam: Elsevier.

Petrides M, Alivisatos B, Evans AC, Meyer E (1993a) Dissociation of human mid-dorsolateral from posterior dorsolateral frontal cortex in memory processing. Proc Natl Acad Sci USA 90:873-877.

Petrides M, Alivisatos B, Meyer E, Evans AC (1993b) Functional activation of the human frontal cortex during the performance of verbal working memory tasks. Proc Natl Acad Sci USA 90:878-882.

Rogers RD, Sahakian BJ, Hodges JR, Polkey CE, Kennard C, Robbins TW (1998) Dissociating executive mechanisms of task control following frontal lobe damage and Parkinson's disease. Brain 121:815-842.

Rogers RD, Andrews TC, Grasby PM, Brooks DJ, Robbins TW (2000) Contrasting cortical and subcortical activations produced by attentional-set shifting and reversal learning in humans. J Cognit Neurosci 12:142-162.

Rolls ET (1994) Neurophysiology and cognitive functions of the striatum. Rev Neurol (Paris) 150:648-660.

Sakashita Y (1991) Visual attentional disturbance with unilateral lesions in the basal ganglia and deep white matter. Ann Neurol 30:673-677.

Stuss DT, Levine B, Alexander MP, Hong J, Palumbo C, Hamer L, Murphy KJ, Izukawa D (2000) Wisconsin Card Sorting Test performance in patients with focal frontal and posterior brain damage: effects of lesion location and test structure on separable cognitive processes. Neuropsychologia 38:388-402.

Talairach J, Tournoux P (1988) Co-planar stereotactic atlas of the human brain. Stuttgart: Thieme.

Taylor SF, Kornblum S, Lauber EJ, Minoshima S, Koeppe RA (1997) Isolation of specific interference processing in the Stroop task: PET activation studies. NeuroImage 6:81-92. 
Toni I, Passingham RE (1999) Prefrontal-basal ganglia pathways are involved in the learning of arbitrary visuomotor associations: a PET study. Exp Brain Res 127:19-32.

Vandenberghe R, Dupont P, De Bruyn B, Bormans G, Michiels J, Mortelmans L, Orban GA (1996) The influence of stimulus location on the brain activation pattern in detection and orientation discrimination. A PET study of visual attention. Brain 119:1263-1276.

Wise SP, Murray EA, Gerfen CR (1996) The frontal cortex-basal ganglia system in primates. Crit Rev Neurobiol 10:317-356.

Worsley KJ, Marrett S, Neelin P, Vandal AC, Friston KJ, Evans AC (1996) A unified statistical approach for determining significant signals in images of cerebral activation. Hum Brain Mapp 4:58-73.
Worsley KJ, Liao C, Grabove M, Petre V, Ha B, Evans AC (2000) A general statistical analysis for fMRI data. NeuroImage 11:S648.

Wright MJ, Burns RJ, Geffen GM, Geffen LB (1990) Covert orientation of visual attention in Parkinson's disease: an impairment in the maintenance of attention. Neuropsychologia 28:151-159.

Yamada T, Izyuuinn M, Schulzer M, Hirayama K (1990) Covert orienting attention in Parkinson's disease. J Neurol Neurosurg Psychiatry 53:593-596.

Zalla T, Koechlin E, Pietrini P, Basso G, Aquino P, Sirigu A, Grafman J (2000) Differential amygdala responses to winning and losing: a functional magnetic resonance imaging study in humans. Eur J Neurosci 12:1764-1770. 